\title{
Paraplegia after intercostal neurolysis with phenol
}

\author{
This article was published in the following Dove Press journal: \\ Journal of Pain Research \\ 19 November 2014 \\ Number of times this article has been viewed
}

\section{Lakshman Gollapalli \\ Rudramanaidu Muppuri \\ Department of Anesthesiology and Pain Medicine, Wayne State University/Detroit Medical Center, Detroit, MI, USA}

Correspondence: Lakshman Gollapalli Department of Anesthesiology, Wayne State University/Detroit Medical Center, 3990 John R Road, Detroit, MI 4820I, USA

Tel + I 3/37457233

Email lgollapa@med.wayne.edu; lakshman.bme@gmail.com

\begin{abstract}
In patients with advanced stages of cancer, severe pain is commonly encountered and is very difficult to treat. It affects the quality of life of the patient and the families involved. Pain can be managed using analgesics and adjuvant therapy. However, studies have shown that at least $10 \%-15 \%$ of patients fail to control pain adequately and will experience severe pain. We discuss the case of a 66-year-old female with metastatic adenoid cystic carcinoma of the left submandibular gland and developed paraplegia following intercostal neurolysis with phenol. After a successful diagnostic T6 to T12 intercostal nerve block, the patient was scheduled for an intercostal neurolytic block. We injected $2 \mathrm{~mL}$ of $10 \%$ aqueous phenol at each level on the left from the T6 to T12 ribs. One hour after the procedure, the patient developed bilateral lower extremity weakness with difficulty moving. A physical examination showed the absence of sensation to pinpricks and vibration from T10 to S5 and an absence of anal sphincter tone and sensation. Magnetic resonance images of the thoracic and lumbar spine showed leptomeningeal metastatic disease and myelitis. We postulate that the paraplegia could be from phenol diffusing along either the spinal nerves or the paravertebral venous plexus into the subarachnoid space. This case report points to the risks involved with phenol neurolysis close to the spine, and we propose alternative methods to minimize neurological complications.
\end{abstract}

Keywords: intercostal neurolysis, pain, phenol, paraplegia

\section{Introduction}

Severe pain is a frequently encountered symptom that affects the quality of life for advanced cancer patients. It has been estimated that $60 \%-90 \%$ of all patients dying of cancer will experience pain in the terminal phase of their disease. ${ }^{1-3}$ Pain can be managed using analgesics and adjuvant therapy titrated according to the World Health Organization's analgesic ladder. ${ }^{4}$

When pain is resistant to standard therapy or when severe side effects of analgesics occur, alternative analgesic techniques should be considered. These include regional nerve blocks, intrathecal analgesic delivery methods, spinal cord stimulation, neurolytic blocks, and vertebroplasty. ${ }^{5}$ Injections of neurolytic agents to destroy nerves and interrupt pain pathways have been used for many years. ${ }^{6}$ In terminally ill cancer patients, phenol has been administered using the intrathecal or epidural routes for the blockade of sympathetic ganglia (celiac, superior hypogastric, ganglion impar, and so on). ${ }^{7}$ Current knowledge and techniques allow these procedures to be performed safely and expeditiously, even though the risk-benefit ratio associated with neurolysis techniques is narrow. The use of phenol for neuroablation for chronic malignant pain is widely accepted, especially when the life expectancy is low. We discuss a case of 
paraplegia followed by phenol intercostal neurolysis for intractable intercostal neuralgia.

\section{Materials and methods}

A 66-year-old Caucasian female was diagnosed with adenoid cystic carcinoma of the left submandibular gland in 1997. She had a left radical neck dissection, radiation, and chemotherapy. In 2005, the patient was diagnosed with extensive metastases to the pleura, lungs, breast, spleen, retroperitoneum, and bones. Since 2005, she had multiple thoracenteses and radiation therapy. The patient complained of lancinating pain in the left side of the chest, was unresponsive to medical therapy, and was referred to the pain clinic for interventional management by the oncology care team. Medical causes of chest pain were ruled out before diagnostic intercostal nerve blocks were scheduled. She had no significant medical problems other than constipation from opioids, and she had a 20 pack-year smoking history.

The patient was brought to the operating room and was placed in a prone position. Standard American Society of Anesthesiologists monitoring was applied, and the patient received anesthesia with an intravenous midazolam, fentanyl, and propofol infusion. The patient's back was prepared and draped in a sterile fashion. We had decided to proceed with an intercostal neurolytic block when the patient reported $60 \%-70 \%$ pain relief for about $6-8$ hours with a diagnostic T6 to T12 intercostal nerve block. The left T6 to T12 ribs were identified 6-7 cm from the midline and marked. The inferior approach was used to insert a 1.5-inch, 25 gauge needle into the inferior border of each rib; the needle was advanced until the periosteum was contacted (Figure 1). The needle tip was walked off the inferior margin of the rib and advanced 0.5 $\mathrm{cm}$. After negative aspiration, $1 \mathrm{~mL}$ of iopamidol dye was injected, and adequate horizontal spread was seen along the inferior border. At each level, $2 \mathrm{~mL}$ of $10 \%$ aqueous phenol was injected with ease. The patient was sedated well and did not respond to any verbal commands. No intraoperative complications were noted.

One hour after the procedure, the patient was awake and had stable vital signs. However, she had bilateral lower extremity weakness with difficulty moving. A physical examination showed an absence of sensation to pinpricks and vibration from $\mathrm{T} 10$ to S5, no voluntary anal contraction, and an absence of anal sensation. Her bilateral lower extremity muscle strength was $0 / 5$, and she showed an absence of deep tendon reflexes. Intravenous methylprednisolone was given, as per the National Acute Spinal Cord Injury Study III guidelines.

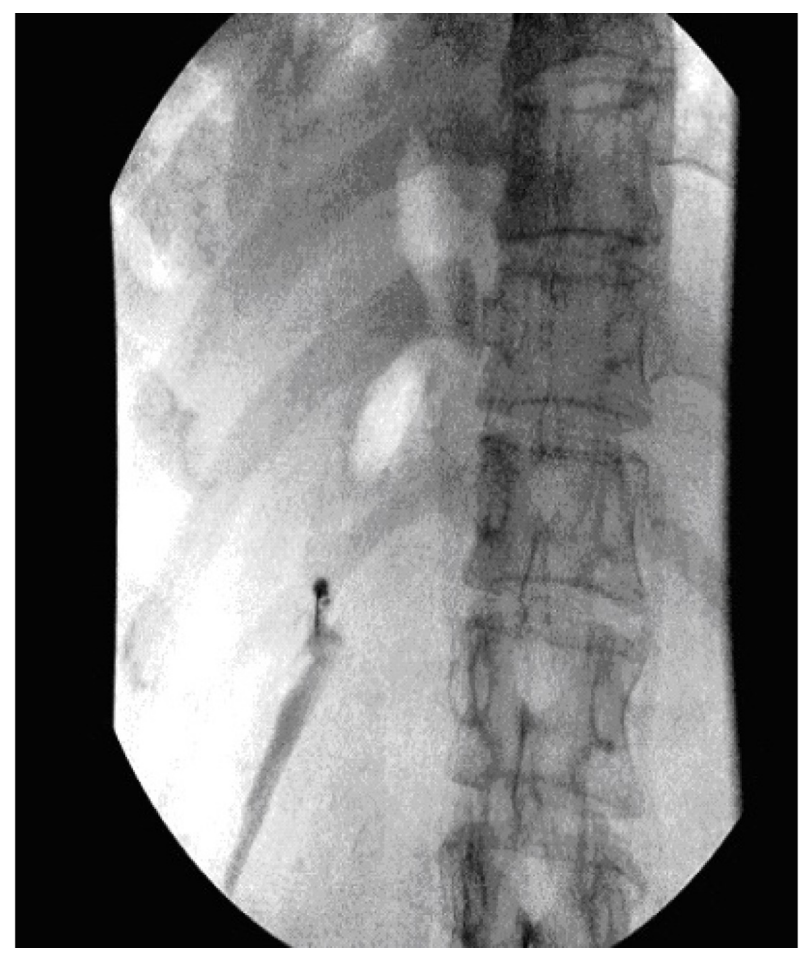

Figure I Fluoroscopic view of horizontal spread of contrast dye, prone position. Note: The needle tip is seen near the inferior border of TI 2 rib on the left.

The patient was admitted, and a neurosurgeon and a neurologist were consulted. Magnetic resonance imaging of the thorax and lumbar spine showed extensive bony metastasis, leptomeningeal metastatic disease, and myelitis at T6 and below (Figure 2). No surgical intervention was recommended, and she was referred for physical medicine and rehabilitation. There was neurogenic bladder dysfunction requiring intermittent catheterization; rectal emptying had to be regulated by laxatives. No clinical remission was seen even after 6 months of rehabilitation; hence, the neurological deficits were considered persistent.

\section{Discussion}

Poorly controlled pain can be devastating and can severely impair quality of life and activities of daily living. ${ }^{3}$ Studies have shown that at least $10 \%-15 \%$ of patients fail to control pain adequately and will experience severe pain in spite of analgesic therapy. ${ }^{5}$ As many as $46 \%$ of terminal patients receive inadequate pain treatment, as reported by family members. $^{8}$

Neurolysis of the intercostal nerve appears to be a costeffective approach to treating intercostal neuralgia associated with cancer. The benefits of intercostal neurolysis include improved analgesia, reduced opioid consumption, and superior clinical effects, due to the absence of deleterious 


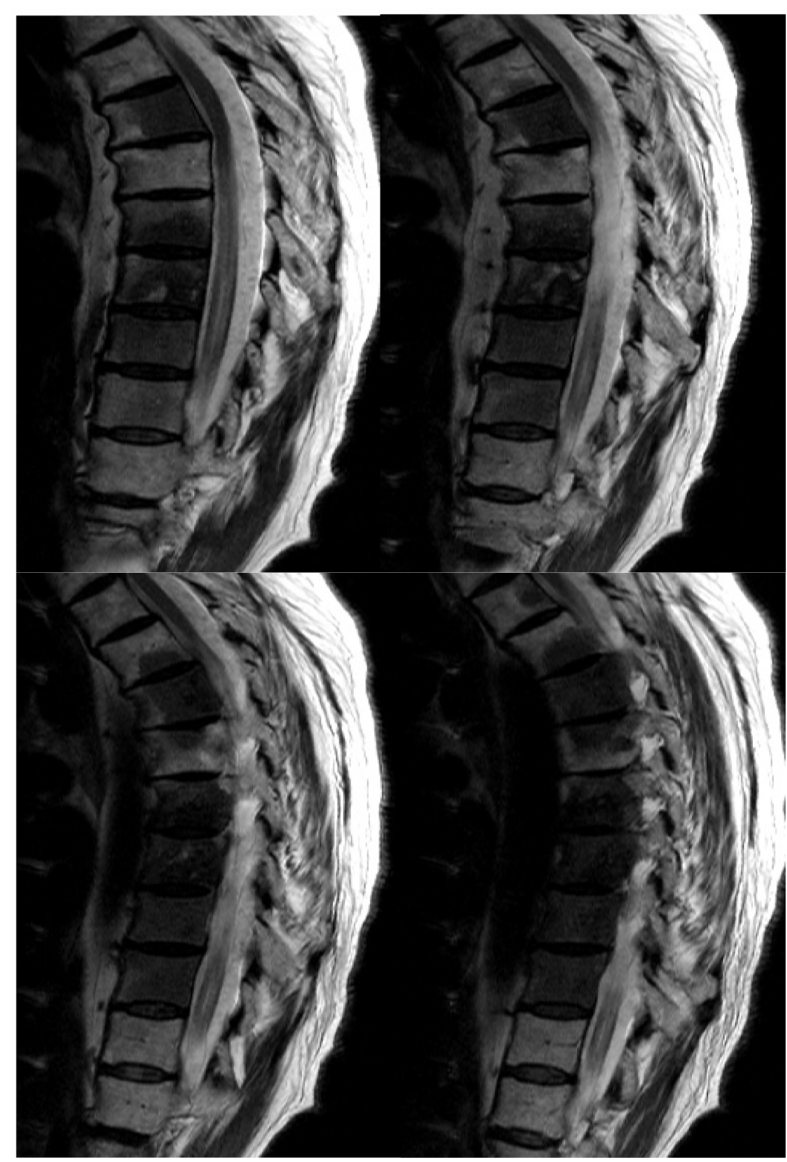

Figure 2 Magnetic resonance images of the thoracic spine, sagittal view, different sections, showing the thoracic body involvement.

Note: Extensive metastatic disease of the vertebral body can be seen.

properties of high-dose chronic opioid therapy. Pain practitioners have been performing these blocks as a part of adjuvant therapy for the optimal treatment of cancer pain.

Phenol, also known as carbolic acid and as hydroxybenzene, was introduced in the 1950s. ${ }^{9}$ It is a colorless, crystalline substance that has a characteristic odor and is soluble in water and organic solvents. It has been in use for neuroablation in chronic cancer pain for a long time. However, because of the complications that phenol causes, its use for chemical neurolysis for chronic noncancer pain is less common. When injected near motor nerves, phenol can produce flaccid paralysis and might also cause systemic complications, such as nausea and vomiting, central nervous system stimulation, cardiovascular depression, and cardiac arrhythmias. ${ }^{10}$ Phenol can diffuse from the paravertebral gutter through the intervertebral foramina toward the epidural space, and then to the cerebrospinal fluid to cause persistent paraplegia. ${ }^{11}$

Invasive use of phenol has been shown to cause irreversible neural tissue damage, and several complications have been reported. ${ }^{10,12}$ There was one case report in which a patient had persistent paraplegia following an intercostal block with a $7.5 \%$ aqueous phenol solution. ${ }^{11}$

In our case, the patient could have developed paraplegia from the diffusion of the phenol along the spinal nerves or the paravertebral venous plexus into the subarachnoid space. It is unlikely to be intravascular, since the symptoms presented about an hour after the procedure. There is a possibility that rapid infusion of the drug could have forced the solution to diffuse faster into the spinal space, even though only $2 \mathrm{~mL}$ of phenol had been used. Paraplegia could also occur from vertebral or lymph node metastases or though direct extension from adjacent primary tumors, such as lung carcinoma, or through leptomeninges, thereby damaging the motor and sensory roots in leptomeningeal metastatic disease. The phenol intrathecal elimination half-life is 8 minutes, as revealed from the Renografin ${ }^{\circledR}$ solution in monkeys. ${ }^{7}$ Slow diffusion of phenol through the leptomeninges at multiple levels leads to neurological damage. Deep sedation masked the immediate effects of neurological damage in our case. Leptomeningeal carcinomatosis that involves the spinal nerves often has a nodular appearance in which tumor foci appear as a string of beads throughout the cauda equina and extend out of the nerves into the neural foramina. Along the spinal cord, a leptomeningeal tumor typically presents as a thin coating along the pia mater, although nodularity and diffuse involvement of the arachnoid space also can occur.

These are some alternative methods to minimize the neurological complications with intercostal neurolysis in leptomeningeal metastatic disease:

1. Especially for the blockade of peripheral nerves near the spine, lipid phenol should be used instead of aqueous solutions, because diffusion and unwanted damage cannot be excluded reliably. ${ }^{11}$ (The use of phenol solutions in water may result in 50 times higher toxicity than that of lipid phenol.) $)^{13}$

2. For leptomeningeal metastatic disease, radiofrequency neuroablation is an alternative treatment method to phenol for intercostal neurolysis, which is used in the treatment of the most common chronic nonmalignant pain syndromes. ${ }^{14}$

3. Intercostal neurolysis should be performed at the midaxillary line, ${ }^{15,16}$ far from the costovertebral joint.

4. Mild sedation with patient communication during the intercostal neurolysis procedure minimizes the chance of developing permanent neurological damage. 


\section{Conclusion}

This case highlights the risk associated with aqueous phenol application in the vicinity of the spinal cord for leptomeningeal metastatic disease. We recommend that patient selection prior to the interventional pain treatment is very important. We also recommend that alternative methods be used for intercostal neurolysis in leptomeningeal metastatic disease patients whenever possible.

\section{Disclosure}

The authors report no conflicts of interest in this work.

\section{References}

1. Keefe FJ, Abernethy AP, C Campbell L. Psychological approaches to understanding and treating disease-related pain. Annu Rev Psychol. 2005;56:601-630.

2. Portenoy RK. Cancer pain. Epidemiology and syndromes. Cancer. 1989;63(Suppl 11):2298-2307.

3. Christo PJ, Mazloomdoost D. Cancer pain and analgesia. Ann NY Acad Sci. 2008;1138:278-298.

4. Cancer pain relief and palliative care. Report of a WHO Expert Committee. World Health Organ Tech Rep Ser. 1990;804:1-75.

5. Sloan PA. The evolving role of interventional pain management in oncology. J Support Oncol. 2004; 2(6):491-500, 503.
6. Maher RM. Intrathecal chlorocresol in the treatment of pain in cancer. Lancet. 1963;281(7288):965-967.

7. Concilus RR, Sehlhorst CS, Denson DD, Katz J, Gregg RV. Dural transfer of phenol following epidural injection in cynomologus monkeys [abstract]. Anesthesiology. 1988;69(3A):A399.

8. Tolle SW, Tilden VP, Rosenfeld AG, Hickman SE. Family reports of barriers to optimal care of the dying. Nurs Res. 2000;49(6):310-317.

9. Patt RB. Cancer Pain. Philadelphia, PA: Lippincott Williams \& Wilkins; 1993.

10. Superville-Sovak B, Rasminsky M, Finlayson MH. Complications of phenol neurolysis. Arch Neurol. 1975;32(4):226-228.

11. Kowalewski R, Schurch B, Hodler J, Borgeat A. Persistent paraplegia after an aqueous $7.5 \%$ phenol solution to the anterior motor root for intercostal neurolysis: a case report. Arch Phys Med Rehabil. 2002; 83(2):283-285.

12. Galizia EJ, Lahiri SK. Paraplegia following coeliac plexus block with phenol. Case report. Br J Anaesth. 1974;46(7):539-540.

13. Cain HD. Subarachnoid phenol block in the treatment of pain and spasticity. Paraplegia. 1965;3(2):152-160.

14. Van Zundert J, Raj P, Erdine S, van Kleef M. Application of radiofrequency treatment in practical pain management: state of the art. Pain Pract. 2002;2(3):269-278.

15. Scott DB. Techniques of Regional Anesthesia. 2nd ed. Norwalk, CT: Appleton and Lange; 1995.

16. Kreuscher H. Regionale Schmerztherapie. In: Niesel HC, editor. Regionalana"sthesie, Lokalana"sthesie, Regionale Schmerztherapie. Stuttgart, Germany: Georg Thieme; 1994:709-761.
Journal of Pain Research

\section{Publish your work in this journal}

The Journal of Pain Research is an international, peer-reviewed, open access, online journal that welcomes laboratory and clinical findings in the fields of pain research and the prevention and management of pain. Original research, reviews, symposium reports, hypothesis formation and commentaries are all considered for publication.

\section{Dovepress}

The manuscript management system is completely online and includes a very quick and fair peer-review system, which is all easy to use. Visit http://www.dovepress.com/testimonials.php to read real quotes from published authors. 